\title{
EVIDENCIA DE UTILIZACIÓN OPORTUNISTA DE NORMAS CONTABLES EN EL RECONOCIMIENTO DE PASIVOS FISCALES DIFERIDOS EN EMPRESAS BRASILEÑAS Y ESPAÑOLAS
}

\section{EVIDENCE OF OPPORTUNIST UTILIZATION OF ACCOUNTING STANDARDS PROCEDURES ABOUT DEFERRED TAX LIABILITIES RECOGNITION IN BRAZILIAN AND SPANISH COMPANIES}

\author{
Clovis Antonio Kronbauer ; José Moreno Rojas ${ }^{b}$; Ernani Ottc; Marcos Antonio Souza ${ }^{d}$ \\ ${ }^{a}$ Doutor em Contabilidade e Auditoría pela Universidade de Sevilla - Espanha \\ Coordenador Executivo do Curso de Graduação em Cências Contábeis da Universidade do Vale do Rio dos Sinos,
E-mail: clovisk@unisinos.br \\ ${ }^{b}$ Doutor em Ciências Econômicas e Empresariais pela Universidade de Sevilla - Espanha; \\ E-mail: jrojas@us.es \\ c Doutor em Ciências Contábeis pela Universidade de Deusto - Espanha \\ Docente titular da Universidade do Vale do Rio dos Sinos e da Faculdade São Francisco de Assis - UNIFIN; \\ E-mail:ernani@unisinos.br \\ ¿ Professor Titular da Universidade do Vale do Rio dos Sinos, UNISINOS, \\ Doutorado em Controladoria e Contabilidade pela Universidade de São Paulo; São Leopoldo, RS - Brasil. \\ E-mail:marcosas@unisinos.br
}

\section{Resumen}

En este estudio se investiga la relación que pueda existir entre el nivel de reconocimiento de Pasivos Fiscales Diferidos y variables explicativas relacionadas con la situación económica, financiera y el tamaño de las empresas. Este trabajo es parte de una investigación más amplia, y que está sendo desarrollada por profesores brasileños y españoles, y por lo tanto, es base para futuras investigaciones en el tema. La presente investigación se realiza en dos muestras de empresas españolas y brasileñas, durante los años de 2003 hasta 2005, y tendrá continuación futura. El análisis se ha realizado por medio de una regresión múltiple, por mínimos cuadrados ordinarios, con los datos dispuestos en panel. El nivel de pasivos fiscales diferidos ha sido calculado a partir del Ratio de Pasivos fiscales Diferidos (RPFD), y contrastado en la regresión múltiple con las variables explicativas. Los resultados obtenidos revelan que la variación del RPFD en la muestra de empresas del IBOVESPA, es explicada en aproximadamente al 95\% por la variación del ratio del Endeudamiento. En las empresas españolas del IBEX-35, la variación del RPFD, es explicada próximamente al 82\% por la variación de los ratios de Endeudamiento y del Tamaño de la empresa.

Palabras-clave: Pasivos Fiscales; Impuesto sobre Beneficios; Manipulación Contable.

\section{Abstract}

This study investigates the relationship that may exist between the level of accounting recognition of deferred tax liabilities and explanatory variables related to economic, financial and company size. This study is part of a wider investigation - this path developed by Brazilian and Spanish professors, and therefore was performed on two samples of Spanish and Brazilian companies during the year 2003 to 2005 and tends to future continuation. The analysis was performed using a multiple regression using ordinary least square, with data arranged in the panel. The level of deferred tax liabilities has been calculated from the ratio of deferred tax liabilities (DTL), and in multiple regressions with the explanatory variables. The main results show that the DTL variation in the sample companies IBOVESPA during the review period is explained by about $95 \%$ by varying the ratio of indebtedness. In Spanish companies in the IBEX-35 RPFD variation during the period analyzes is soon explained $82 \%$ of the variation in debt ratios and firm size.

Keywords: Tax Liabilities; Tax Benefits; Accounting Manipulation. 


\section{INTRODUCIÓN}

En los últimos años, varios organismos emisores de normas contables, han emitido normas que regulan el tratamiento del Imopuesto sobre Beneficios (IB), buscando garantizar que la inrfromacíon contable no esté distorsionada por la influencia de la normativa fiscal.

Según Moreno (1997, p.11), el objetivo de la contabilidad se pierde o se rompe con relativa frecuencia por la interferencia de la legislación tributaria. Así, surge un problema de gran magnitud cuando normas legales obligan a las empresas a contabilizar ciertas operaciones aplicando criterios o reglas fiscales en contra de los principios contables. En estas ocasiones, la contabilidad produce una información híbrida, por aplicar principios contables en algunas operaciones y criterios y reglas fiscales en otras.

En la opinión de Alonso, Nevado y Nuñes (2003, p. 19), las normas fiscales no deberían regular la contabilidad, pues la base imponible se calcula partiendo de las cuentas anuales elaboradas de acuerdo con los principios contables, pero ajustándose a las normas fiscales. Esto garantiza la independencia entre la contabilidad y la fiscalidad.

Sin embargo, para García-Ayuso y Zamora (2003, p. 397), aunque los gestores de las empresas cumplan con lo que requieren las normas contables, en muchas ocasiones, en el momento de preparar las cuentas anuales, es necesario realizar juicios de valor a la hora de optar por una entre las diferentes alternativas que las normas contables ofrecen. Estas elecciones pueden ser susceptibles de modificar el valor de la composición del patrimonio, condicionando así, la sustancia y el estilo de la información comunicada a los usuarios.

A causa de esta afirmación, es necesario investigar en una muestra de empresas, en este caso, empresas españolas y brasileñas, si el reconocimiento de pasivos fiscales diferidos no podría estar relacionado con elecciones oportunistas por parte de los gestores. Así, el objetivo principal del estudio es identificar la relación que pueda existir entre el nivel de reconocimiento de Pasivos Fiscales Diferidos y variables económicas, financieras y de tamaño de las empresas investigadas. Esta investigación, se desarrolla en dos muestras distintas de empresas, es decir: una muestra de empresas españolas del IBEX-35 y otra muestra de empresas brasileñas del IBOVESPA.

El estudio desarrollado tiene especial relevancia pues presenta un aspecto novedoso en investigaciones cuantitativas que se ocupan del reconocimiento de activos y/o pasivos fiscales diferidos, por el hecho buscar una relación que pueda existir entre el nivel de Pasivos Fiscales Diferidos y variables explicativas escogidas para el análisis. Hasta el momento actual, los estudios de esa naturaleza, se habían ocupado de investigar factores determinantes en el reconocimiento de Activos Fiscales Diferidos (MILLER y SKINNER, 1998; GORDON y JOOS, 2004; WASSERMAN, 2004; KRONBAUER y MORENO, 2008).

Además, es importante señalar que esta investigación es parte de un estudio más amplio, desarrollado por investigadores españoles y brasileños, y que por lo tanto, es una continuación de estudios anteriores y tendrá desarrollos y desdoblamientos posteriores.

\section{FUNDAMENTACIÓN TEÓRICA}

\subsection{Consideración Contable del Impuesto sobre Beneficios y las Diferencias Temporarias}

El cargo por el Impuesto sobre Beneficios, al igual que el resto de partidas de la 
cuenta de resultados, debe ser considerado como un gasto del ejercicio, y en su cálculo debe ser considerando el principio del devengo. Utilizar este principio, implica considerar el gasto por IB calculado sobre la base del resultado contable, y así, pueden surgir diferencias de carácter temporal entre el gasto del impuesto y la provisión, suponiendo un cargo o abono en las respectivas cuentas de Activos y/o Pasivos Fiscales Diferidos (LABATUT, 1992, p. 148).

Las diferencias de carácter temporal, son definidas como aquellas que se originan por el distinto tratamiento contable y fiscal en el reconocimiento y medida de activos, pasivos, ingresos y gastos. Estas diferencias temporarias, tienen su origen en (GALLEGO, 1997, p. 146):

1. Diferencias entre la cantidad reflejada entre la base imponible y el resultado contable en un determinado ejercicio, esto es, estas diferencias se originan cuando ingresos o ganancias, gastos o pérdidas se incluyen en el resultado fiscal con anterioridad o posterioridad a su inclusión el resultado contable; y

2. Diferencias existentes entre el valor de la base fiscal de activos y pasivos y su valor contable, cantidad que se refleja en los estados financieros. Así, la diferencia que exista entre la base fiscal de un activo o pasivo y su importe en libros, dará lugar a importes deducibles o a tributar en años futuros cuando las cantidades registradas de activos sean recuperadas y las de pasivos liquidadas.

Según interpretación de Castillo (2004, p. 178), las diferencias temporarias se clasifican en imponibles y deducibles. Las imponibles son las que en un futuro resultarán en valores imponibles a efectos del Impuesto sobre Beneficios, originando los Pasivos Fiscales Diferidos en el ejercicio en el cual se produzcan. Ya las diferencias temporarias deducibles, son aquellas que resultarán en valores deducibles en el cálculo del resultado imponible de períodos futuros, generando los Activos Fiscales Diferidos en el ejercicio corriente.

\subsection{Pasivos Fiscales Diferidos}

Los Pasivos Fiscales Diferidos, se originan de las diferencias temporarias imponibles, y para que se les pueda caracterizar, es necesario identificar sus aspectos conceptuales.

Según Hendriksen y Van Breda (1999, p.283), los pasivos pueden ser caracterizados como sacrificios futuros probables de beneficios económicos, consecuentes de obligaciones presentes de una determinada entidad, en cuanto a la transferencia de activos o prestación de servicios a otras entidades en el futuro, a consecuencia de sucesos pasados.

Según señala Iudícibus (2006, p. 158), gran parte de las obligaciones está relacionada con el reconocimiento de gastos. Para reconocer un ingreso es necesario tener condiciones para estimar los gastos asociados, aun cuando el pago de éstos ocurra solamente en el futuro, en un período que en el momento actual no se pueda determinar. En estas situaciones se reconocerá un pasivo correspondiente al gasto incurrido.

Basados en las definiciones de pasivo y en los aspectos con él relacionados, es posible identificar los elementos que caracterizan un Pasivo Fiscal Diferido como un pasivo: 
Pasivos Fiscales Diferidos en Empresas Brasileñas y Españolas

- El Pasivo Fiscal Diferido es reconocido por estar relacionado con un gasto por Impuesto sobre Beneficios, y dicho gasto está, por su parte, relacionado con un ingreso, que es el beneficio contable generado en el ejercicio;

- El Pasivo Fiscal Diferido tiene origen en una provisión de gastos por IB, y representa en el momento actual, una deuda de la empresa con la hacienda, y por lo tanto, deberá ser abonada en un futuro; y

- Dicho pasivo está relacionado con sucesos pasados, es decir, beneficios ya obtenidos, y sobre los cuales se ha reconocido un gasto que deberá ser pagado en un futuro.

Así, se concluye que un Pasivo Fiscal Diferido es un pasivo legítimo, representado una deuda de la empresa, y en su reconocimiento debe respetarse las normas contables específicas.

\subsection{Reconocimiento de Pasivos Fiscales Diferidos}

\subsubsection{Determinaciones de Normas Contables de Ámbito Internacional}

Siguiendo los princípios contables de devengo y prudencia, las principales normativas contables en el ámbito internacional, requieren el reconocimiento de Pasivos Fiscales Diferidos para todas las diferencias temporarias imponibles.

El SFAS n ${ }^{\circ} 109$ del FASB establece que un Pasivo Fiscal Diferido será reconocido en función de todas las diferencias temporarias imponibles. La medición de los estará basada en lo que establece la legislación tributaria en vigor, mientras que los efectos de los futuros cambios en la legislación o en los tipos impositivos no serán aplicados por anticipación.

Ya el IASB, establece en la Norma Internacional de Contabilidad $n^{\circ} 12$, que la empresa reconocerá un Pasivo Fiscal Diferido por causa de cualquier diferencia temporaria imponible, a menos que la diferencia haya surgido por una condición por la que esta norma no permita tal reconocimiento. Estos pasivos deben medirse según tipos impositivos que vayan a ser de aplicación en los períodos en los que se espere pagarlos, a partir de la normativa y tipos impositivos que se hayan aprobado, o estén a punto de aprobarse, en la fecha del balance.

\subsubsection{La Normativa Contable Española}

La actual normativa que regula el tratamiento contable del Impuesto sobre Sociedades en España, está prevista en la Norma de Valoración $13^{\text {a }}$ del nuevo Plan General de Contabilidad (PGC) de 2007, aprobado por el Real Decreto 1514/2007, de 16 de noviembre, y tiene vigencia a partir del ejercicio financiero de 2008.

Sin embargo, durante el período relativo a esta investigación, las normas que regulaban el tratamiento contable del Impuesto sobre Beneficios eran la Norma de Valoración $16^{\mathrm{a}}$ del PGC de 1990, y las Resoluciones del Instituto de Contabilidad y Auditoría de Cuentas (ICAC), de 9 de octubre de 1997 y de 15 de marzo de 2002. Según requieren estas normas, por aplicación del principio de prudencia, los pasivos fiscales diferidos deberán ser objeto de contabilización en todo caso, y su importe vendrá determinado por la aplicación del tipo de gravamen del ejercicio 
sobre la diferencia, para cada operación, entre la base imponible y el resultado contable antes de impuestos que revierte en períodos futuros.

\subsubsection{Las Normas Contables Brasileñas}

Las normas contables brasileñas actuales, que regulan el tratamiento contable del Impuesto sobre Beneficios tiene su origen en el pronunciamiento técnico del Comité de Pronunciamientos Contables (CPC), es decir, el CPC n 32 - Tributos Sobre o Lucro, que tiene correlación con la Norma Internacional de Contabilidad 12 del IASB. Sin embargo, las normas que tenían vigencia para el período de este estudio son: la Deliberación $n^{0}$ 273/1998 de la Comisión de Valores Mobiliarios (CVM) - Impuesto sobre la Renta de la Persona Jurídica y Contribución Social sobre el Beneficio Neto; y, la Norma Brasileña de Contabilidad - Técnica (NBC T) 19.2 - Tributos sobre Beneficios, según Resolución nº 998/2004, del Consejo Federal de Contabilidad (CFC).

Según estas dos normas de la CVM y del CFC, a causa de las diferencias temporarias imponibles, surgen los Pasivos Fiscales Diferidos, o simplemente pasivos postergados. Como consecuencia de estas diferencias, las empresas reconocerán estas obligaciones diferidas en el Pasivo al mismo tiempo en que se añade al gasto con los tributos sobre beneficios, el valor correspondiente en el estado de resultados.

Estos pasivos deben ser reconocidos según los tipos impositivos aplicables al período en el que el pasivo será liquidado. Cuando se aplican diferentes tipos a los diversos niveles de beneficio imponible, los pasivos fiscales deben ser reconocidos por los tipos medios debiendo haber revisión periódica de su valor.

\subsection{Estudios Anteriores Relativos al Reconocimiento de Activos/Pasivos Fiscales Diferidos}

Las elecciones llevadas a cabo en el momento de aplicar las normas contables, podrían llevar a los directores de las empresas a utilizar la discrecionalidad que estas normas les confieren para modificar el resultado y la composición del patrimonio. Sirve como ejemplo la decisión de reconocer (o no) activos fiscales diferidos, pues la consecuencia de dicho reconocimiento puede generar una reducción en el ratio de endeudamiento, a causa del aumento del valor del activo y de la mejora de los fondos propios. Puede deducirse que un elevado nivel de endeudamiento, podrá aumentar la propensión de la empresa en registrar activos fiscales diferidos, utilizando de manera oportunista las alternativas conferidas por las normas contables (MILLER y SKINNER, 1998, p. 217; GORDON y JOOS, 2004, p. 102).

Investigando la decisión en reconocer créditos de naturaleza fiscal, García-Ayuso y Zamora (2003) concluyeron que el nivel de beneficios pasados y futuros de las empresas está positivamente relacionado con la probabilidad del reconocimiento. La posible mejora de los ratios de endeudamiento que el reconocimiento de estos créditos conlleva también parece ser un factor determinante de la decisión de activar el crédito, existiendo una asociación positiva en la relación de estas variables.

Wassermann (2004), investigando las instituciones bancarias brasileñas sobre el reconocimiento y contabilización de activos fiscales diferidos, pudo comprobar que a partir del 
año 1997, cuanto aumentaran las diferencias entre las normativas contable y fiscal, principalmente en lo que se refiere a las provisiones no deducibles, hubo un crecimiento continuado en el valor de los activos fiscales diferidos en el conjunto de dichas instituciones.

Investigando una muestra de empresas brasileñas, Kronbauery Alves (2008), concluyeron que en las mismas, el reconocimiento de Activos Fiscales Diferidos, durante el período analizado, es explicado próximamente al $93 \%$ por la variación de los ratios de Endeudamiento y Tamaño (según el valor del Activo de las empresas). Los resultados revelan que los gestores de esta empresas puedan estar reconociendo un valor mayor de Activos Fiscales Diferidos cuando el ratio Endeudamiento estuviera mayor, buscando así, aumentar el valor del activo, mejorando los Fondos Propios por el reflejo de un mejor resultado, y como consecuencia, disminuir el ratio de Endeudamiento.

Moreno et al (2009), investigando una muestra de empresas españolas, han concluido que las empresas con mayor nivel de endeudamiento presentan mayores valores del índice de diferencias temporales positivas, lo cual sugiere que un endeudamiento alto aumenta la propensión en reconocer activos fiscales diferidos. Igualmente, en este estudio, las empresas de la muestra con menor nivel de liquidez presentaran valores mayores del índice de diferencias temporales positivas, lo que indica que las empresas con baja o media liquidez presentan una mayor propensión para la activación de impuestos anticipados.

Considerando las afirmaciones presentadas por autores y las conclusiones obtenidas en estudios anteriores, en esta investigación se buscará identificar si el reconocimiento de Pasivos Fiscales Diferidos también no puede estar relacionado con elecciones oportunistas por parte de los gestores y responsables por la preparación de los estados financieros.

\section{ASPECTOS METODOLÓGICOS DEL ESTUDIO}

\subsection{Clasificación de la Investigación}

Por su naturaleza, el presente estudio puede ser caracterizado como una investigación aplicada, pues, según Marconi y Lakatos (1996, p. 19), dichas investigaciones se caracterizan por su interés práctico y sus resultados pueden ser utilizados para la comprensión y resolución de problemas que ocurren en la realidad.

En lo que se refiere a sus objetivos, la presente investigación asume características explicativas, pues busca comprobar la existencia de alguna relación entre las variables que reflejan el nivel de reconocimiento de pasivos fiscales diferidos y variables como el endeudamiento, la liquidez, la rentabilidad y el tamaño de las compañías investigadas.

Considerando la forma de resolución del problema, la presente investigación presenta características cuantitativas. Los métodos cuantitativos presentan mejores resultados cuando se orientan en la búsqueda de asociaciones y explicaciones entre variables, estando directamente relacionados con la predicción de modelos propuestos (OPPENHEIM, 1993, p. 21).

Con respecto a los procedimientos técnicos que se utilizan en el estudio, en lo que se refiere a la obtención de datos, la presente investigación tiene una característica documental.

\subsection{Población, Muestra y Período de Investigación}


La población de empresas españolas que se investiga, se compone de las empresas que tienen sus acciones admitidas para negociación en la Bolsa de Madrid y que componían su índice más importante, el IBEX-35, durante los años 2003 hasta 2005.

La población de empresas brasileñas, se compone de las empresas que tienen sus acciones admitidas para negociación en la Bolsa de Valores de São Paulo-BOVESPA y que componían su índice más importante, el IBOVESPA, durante los años 2003 hasta 2005.

El criterio para la definición de las muestras estudiadas ha sido escogido de manera intencional. Para que las dos muestras presentasen mayor homogeneidad con respecto a las características de las empresas, ha sido necesario quitar del conjunto inicial las instituciones financieras y bancarias, las empresas aseguradoras y otras empresas cuyas cuantas anuales individuales presentasen características muy distintas de la muestra final escogida, como es el caso de empresas que ejercen exclusivamente actividades de control y gestión de inversiones.

Así, cada muestra se compone de 29 empresas, y en consecuencia, han sido objeto del análisis, 87 cuentas anuales individuales en cada muestra. El número de individuos investigados puede ser considerado adecuado y significativo, pues analizar del contenido de un conjunto de 174 cuentas anuales es una tarea que exige del investigador un trabajo de mucha dedicación y de un período de tiempo considerable.

\subsection{Obtención y Clasificación de los Datos}

Los datos para la realización del estudio, han sido obtenidos a partir de las cuentas anuales individuales de las empresas investigadas.

Los estados financieros de la muestra de empresas brasileñas, se han obtenido a partir de la búsqueda en la página web de la Bolsa de São Paulo, mientras que las cuentas anuales de la muestra de empresas españolas han sido obtenidas a partir de las páginas web de la Bolsa de Madrid, de la Comisión Nacional del Mercado de Valores de España.

Para identificar la relación que pueda existir entre el nivel de reconocimiento de pasivos fiscales diferidos y las variables explicativas, a través del análisis econométrico, se han calculado los ratios de las variables a partir de los datos obtenidos de las cuentas anuales de las empresas estudiadas. Estos ratios son identificados en la sección siguiente.

\subsection{Descripción de los Procedimientos Estadísticos}

\subsubsection{Aspectos Generales}

Para buscar una explicación sobre la relación que pueda existir entre las variables analizadas, se ha propuesto un modelo econométrico general, explicado por Wooldridge (2006, p. 11), y basado en todas las variables estudiadas. A continuación, a partir de las pruebas realizadas con la utilización del programa de análisis econométrico EViews (Econometric Views), se ha identificado las variables que pudiesen explicar la variación de la variable dependiente, esto es, el Ratio de Pasivos Fiscales Diferidos (RPFD).

El método de estimación del modelo se define como una regresión múltiple, con datos de panel, relativos al período de 2003 hasta 2005. Dicha regresión ha sido estimada por el 
método de coeficientes fijos. Según Hill et al (2003, p.167), la utilización de datos de panel es la más indicada para las variables analizadas en la presente investigación y la regresión múltiple por mínimos cuadrados ordinarios también esta adecuada para estas situaciones.

El nivel de Pasivos Fiscales Diferidos se obtuvo a través del ratio RPFD, que es el resultado de la división del valor de dichos pasivos por el valor del Pasivo (Activo) Total del balance. En este contexto, se busca entender si la variación del ratio RPFD de la muestra de empresas del IBOVESPA y del IBEX-35, puede ser explicada por el Endeudamiento, la Liquidez Corriente, la Liquidez General, la Rentabilidad Financiera, y el tamaño de la empresa, determinado a partir de los Ingresos Netos Anuales y por el valor del Activo.

\subsubsection{Definición del Modelo Econométrico General y de las Hipótesis}

Para explicar la posible relación entre las variables se ha propuesto un modelo econométrico general con la inclusión de todas las variables explicativas. Con la estimación del modelo se pretende identificar aquellas variables que pueden explicar la variación de la variable dependiente, esto es, el RPFD. E1 Modelo Econométrico General es:

$$
\operatorname{RPFD}_{\mathrm{it}}=\beta_{0}+\beta_{1} \mathrm{ENDTO}_{\mathrm{it}}+\beta_{2} \mathrm{LC}_{\mathrm{it}}+\beta_{3} \mathrm{LG}_{\mathrm{it}}+\beta_{4} \mathrm{RF}_{\mathrm{it}}+\beta_{5} \mathrm{~T}_{\mathrm{it}}+\beta_{6} \mathrm{~T}_{\mathrm{it}}+\alpha_{\mathrm{i}}+\mathrm{e}_{\mathrm{it}}
$$

Los términos del modelo son los siguientes:

- RPFD $_{\mathrm{it}}$ : Ratio de los Pasivos Fiscales Diferidos, de la empresa “i” en el período " $t$ ", que es la variable dependiente, donde:

1. $i=$ empresa $i, p / i \in i=1$ hasta 29 ,

2. $\mathrm{t}=$ años de 2003 hasta 2005 .

- $\beta_{0}$ : Es el término constante del modelo econométrico;

- $\quad \beta_{1} \ldots . . . \beta_{6}$ : Son todas las variables explicativas, parámetros que serán estimados por la regresión múltiple y que pueden explicar la variación del ratio RPFD;

- ENDTO - Endeudamiento calculado para la empresa “i” en el período “t”, obtenido del cálculo: Pasivo Exigible $\div$ Activo Total;

- LC - Liquidez Circulante calculada para la empresa " $i$ ” en el período "t", obtenido de la ecuación Activo Circulante $\div$ Pasivo Circulante;

- LG - Liquidez General calculada para la empresa “i”" en el período “t”, determinado por el cálculo: Activo Realizable $\div$ Deudas Totales

- RF: Rentabilidad Financiera calculada para la empresa "i” en el período "tt", obtenida del cálculo: Resultado Neto $\div$ Fondos Propios; 
- T1: Tamaño 1 calculado para la empresa "i" en el período "t", obtenido del logaritmo del valor neto de la Cifra de Negocios (Ingresos Netos en Brasil);

- T2: Tamaño 2 calculado para la empresa "i" en el período " $\mathrm{t}$ ", obtenido del logaritmo del valor del Activo Total;

- $\quad \alpha_{i}$ : Es la variable no observada y se supone que esté relacionada con las variables independientes; $\mathrm{y}$

- $\mathrm{e}_{\mathrm{it}}$ : Es el término que representa el error de la estimación.

Para contrastar las variables, se han formulado las siguientes hipótesis:

Hipótesis $1\left(\mathrm{H}_{0,1}\right)$ : $\quad$ No existe relación entre el RPFD y el endeudamiento (ENDTO) de las empresas.

Hipótesis $1\left(\mathrm{H}_{1,1}\right)$ : $\quad$ Existe relación entre el RPFD y el endeudamiento de las empresas.

En esa hipótesis se esperaba que la relación entre las variables sea $\beta_{1}<0$, pues los directivos de las empresas puedan tener propensión a reconocer un menor valor de Pasivos Fiscales Diferidos cuando las empresas presenten un ratio de endeudamiento más elevado, como forma de no aumentar el valor de su pasivo.

Hipótesis $2\left(\mathrm{H}_{0,2}\right)$ : No existe relación entre el RPFD y la Liquidez Circulante (LC) de las empresas.

Hipótesis $2\left(\mathrm{H}_{1,2}\right)$ : Existe relación entre el RPFD y la Liquidez Circulante (LC) de las empresas.

En la hipótesis 2, se espera que la relación entre LC y RPFD sea $\beta_{2}>0$. Se supone que las empresas tendrían una propensión mayor para reconocer Pasivos Fiscales Diferidos cuando tuviesen una liquidez al menos aceptable, hecho que no perjudicaría este ratio.

Hipótesis $3\left(\mathrm{H}_{0,3}\right)$ : No existe relación entre el RPFD y la Liquidez General (LG) de las empresas.

Hipótesis $3\left(\mathrm{H}_{1,3}\right)$ : Existe relación entre el RPFD y la Liquidez General (LG) de las empresas.

La relación esperada en esta hipótesis es $\beta_{3}>0$, pues se entiende que los directores de las empresas tendrían mayor propensión en reconocer un valor más significativo de Pasivos Fiscales Diferidos cuando esto no perjudique su ratio de Liquidez General.

Hipótesis $4\left(\mathrm{H}_{0,4}\right)$ : $\quad$ No existe relación entre el RPFD y la Rentabilidad Financiera (RF) de las empresas.

Hipótesis $4\left(\mathrm{H}_{1,4}\right)$ : Existe relación entre el RPFD y la Rentabilidad Financiera (RF) de las empresas..

En la hipótesis 4 , la relación esperada entre las variables es $\beta_{4}>0$, una vez que el reconocimiento de Pasivos Fiscales Diferidos tiene como contrapartida el reconocimiento de un gasto por Impuesto sobre Beneficios, hecho que disminuye el beneficio, o entonces, aumenta 
la pérdida. Así, los gestores podrían tener mayor propensión para reconocer dichos pasivos, cuando el beneficio y la Rentabilidad Financiera estuvieren en niveles favorables.

Hipótesis $5\left(\mathrm{H}_{0,5}\right)$ : No existe relación entre el RPFD y el Tamaño 1 (T1) de las empresas.

Hipótesis $5\left(\mathrm{H}_{1,5}\right)$ : Existe relación entre el RPFD y el Tamaño 1 (T1) de las empresas.

La relación esperada en la hipótesis es $\beta_{5} \neq 0$, pues es difícil imaginar que empresas con mayor o menor valor de ingresos presenten mayor o menor nivel de Pasivos Fiscales Diferidos.

Hipótesis $6\left(\mathrm{H}_{0,6}\right)$ : $\quad$ No existe relación entre el RPFD y el Tamaño 2 (T2) de las empresas.

Hipótesis $6\left(\mathrm{H}_{1,6}\right)$ : $\quad$ Existe relación entre el RPFD y el Tamaño 2 (T2) de las empresas.

La relación esperada en la hipótesis actual es $\beta_{6} \neq 0$, pues no es posible preestablecer una tendencia de que el valor del Activo determine el nivel de Pasivos Fiscales Diferidos.

Presentados los aspectos metodológicos y conocido el modelo econométrico y considerando las hipótesis que serán contrastadas, a continuación se presentan los resultados obtenidos a partir del análisis realizado.

\section{ANÁLISIS DE LOS RESULTADOS DE LA INVESTIGACIÓN}

\subsection{Análisis de la Relación entre el RPFD y las Variables Explicativas en la Muestra de Empresas Brasileñas del IBOVESPA}

A partir de los datos obtenidos de los estados financieros de las empresas de la muestra IBOVESPA, se han calculado los ratios cuyas características básicas se presentan en la Tabla 1, por medio de la estadística descriptiva de las variables analizadas.

Según se presenta en la Tabla 1, la variable dependiente, el RPFD, no se caracteriza como una distribución normal. Además, se identifica una significativa diferencia entre los niveles de pasivos fiscales diferidos a partir de los valores mínimos, máximos y medianos de la variable RPFD, lo que sugiere la adopción de prácticas contables distintas en las empresas.

De las variables explicativas, el ENDTO es una variable que puede ser considerada como una distribución normal, con una probabilidad aproximada de 0,9077 , y con relación a la variable T2, no se puede rechazar la hipótesis de su normalidad en función de la probabilidad de 0,1362 , que es relevante por estar por encima de 0,10 . La no normalidad de las demás variables no impide su utilización para el análisis y la estimación del modelo final.

Conocidos los datos de la estadística descriptiva del conjunto de variables estudiadas, se presenta el análisis preliminar de la relación que puede existir entre el RPFD y las variables explicativas en la muestra IBOVESPA, por medio de la estimación de una regresión múltiple por mínimos cuadrados ordinarios, con los datos agrupados en panel, procediendo la estimación por efecto fijo. En la Tabla 2, se presenta el resultado obtenido. 
Tabla 1: Estadística Descriptiva I - Variables de la Muestra de Empresas del IBOVESPA

\begin{tabular}{|c|c|c|c|c|c|c|c|}
\hline $\begin{array}{c}\text { Descriptive } \\
\text { Statistic }\end{array}$ & RPFD & ENDTO & LC & LG & RF & T1 & T2 \\
\hline Mean & 0,013465 & 0,528699 & 1,163259 & 0,783247 & 0,217025 & 6,605112 & 6,960231 \\
\hline Median & 0,003757 & 0,539135 & 1,003938 & 0,65886 & 0,216153 & 6,611018 & 6,938366 \\
\hline Maximum & 0,106278 & 1,116932 & 5,045733 & 3,20016 & 1,650475 & 8,024578 & 8,187558 \\
\hline Minimum & 0 & 0,09023 & 0,031767 & 0,132173 & $-0,940066$ & 4,675026 & 6,034324 \\
\hline Std. Dev. & 0,023051 & 0,218596 & 0,704324 & 0,503002 & 0,254004 & 0,510417 & 0,469147 \\
\hline Skewness & 2,539002 & 0,112567 & 2,234839 & 2,045017 & 1,03192 & $-0,276699$ & 0,523773 \\
\hline Kurtosis & 9,292509 & 3,052055 & 1,239816 & 9,381096 & 1,788795 & 5,158368 & 3,049 \\
\hline Jarque-Bera & 237,009 & 0,193556 & 392,5998 & 208,2445 & 776,3304 & 17,99741 & 3,986606 \\
\hline Probability & 0 & 0,907757 & 0 & 0 & 0 & 0,000124 & 0,136245 \\
\hline Observations & 87 & 87 & 87 & 87 & 87 & 87 & 87 \\
\hline
\end{tabular}

Observando la Tabla 2, es posible verificar que el modelo econométrico general, presenta un coeficiente de determinación ( $\mathrm{R}^{2}$ ajustado) igual a 0,951191 , esto es, la variación de la variable dependiente (RPFD), es explicada próximamente al 95,1\% por el conjunto de las variables explicativas del modelo. Sin embargo, de las seis variables explicativas, solamente la variable ENDTO presenta real capacidad de explicación, una vez que la probabilidad de no explicación (rechazo) en el modelo es 0,0213. Realizando nueva estimación del modelo, y quitando las variables con menor poder de predicción, al final se comprobó que la única variable que puede explicar la variación del RPFD es el Endeudamiento.

Tabla 2: Regresión múltiple del RPFD y todas las variables explicativas de la muestra IBOVESPA

\begin{tabular}{l|c|c|c|c}
\hline \multicolumn{5}{c}{ Dependent Variable: RPFD } \\
\hline $\begin{array}{c}\text { Method: Panel Least Squares } \\
\text { Cross-sections included: } 29\end{array}$ & \multicolumn{3}{l}{ Sample: 2003 2005 } \\
\hline Variable & Coefficient & Std. Error & t-Statistic & Prob. \\
\hline ENDTO & -0.026483 & 0.011150 & -2.375152 & 0.0213 \\
\hline LC & -0.000843 & 0.001523 & -0.553312 & 0.5824 \\
\hline LG & -0.002398 & 0.005345 & -0.448637 & 0.6556 \\
\hline RF & -0.001206 & 0.003895 & -0.309557 & 0.7581 \\
\hline T1 & -0.006801 & 0.007783 & -0.873872 & 0.3862 \\
\hline T2 & 0.002362 & 0.014768 & 0.159933 & 0.8736 \\
\hline R-squared & 0.059070 & 0.077731 & 0.759927 & 0.4507 \\
\hline Adjusted R-squared & Cross-section fixed (dummy variables) & \\
\hline Durbin-Watson stat & 0.970488 & F-statistic & 50.29321 \\
\hline & 0.951191 & Prob(F-statistic) & 0.000000 \\
\hline
\end{tabular}


La conclusión inicial que se obtiene a partir de las estimaciones realizadas, es que deben aceptarse las hipótesis nulas $\mathrm{H}_{0.2}, \mathrm{H}_{0.3}, \mathrm{H}_{0.4}, \mathrm{H}_{0.5}$, y $\mathrm{H}_{0.6}$. Por consecuencia, se concluye que en el período analizado, la variación del Ratio de los Pasivos Fiscales Diferidos (RPFD) de las empresas del IBOVESPA, no tiene relación significativa con la variación de los ratios de Liquidez Circulante (LC), Liquidez General (LG), Retorno Financiero (RF), ni con el tamaño de las empresa (ingresos netos anuales - T1 y el valor de sus activos - T2).

Para la estimación final del modelo y de la ecuación que explica la variación del RPFD en la muestra IBOVESPA, se mantuvo solamente la variable explicativa ENDTO. En la Tabla 3 , se puede identificar el resultado obtenido con la nueva estimación del modelo.

Tabla 3: Regresión múltiple del RPFD y el ENDTO en la muestra del IBOVESPA

\begin{tabular}{|c|c|c|c|c|}
\hline \multicolumn{5}{|c|}{ Dependent Variable: RPFD } \\
\hline \multicolumn{2}{|c|}{ Method: Panel Least Squares } & \multicolumn{3}{|c|}{ Sample: 20032005} \\
\hline \multicolumn{2}{|c|}{ Cross-sections included: 29} & \multicolumn{3}{|c|}{ Total panel (balanced) observations: 87} \\
\hline Variable & Coefficient & Std. Error & t-Statistic & Prob. \\
\hline ENDTO & -0.018995 & 0.008474 & -2.241516 & 0.0289 \\
\hline $\mathrm{C}$ & 0.023507 & 0.004512 & 5.210255 & 0.0000 \\
\hline \multicolumn{5}{|c|}{ Cross-section fixed (dummy variables) } \\
\hline R-squared & 0.969377 & F-statistic & & 62.21835 \\
\hline Adjusted R-squared & 0.953797 & Prob(F-stati & & 0.000000 \\
\hline Durbin-Watson stat & 2.369109 & & & \\
\hline
\end{tabular}

Observando los resultados de la nueva estimación en la Tabla 3, es posible concluir que la variación del RPFD es explicada aproximadamente al 95,4\% por la variación de la variable explicativa ENDTO. Esto se puede comprobar por el $\mathrm{R}^{2}$ ajustado de 0,953796 y por la probabilidad de rechazo de su influencia sobre la variable dependiente, que está muy por debajo del 0,1 y muy cercana al cero, esto es, 0,0289 en el caso de la variable ENDTO.

Comparándose los datos de la Tabla 3 con los de la Tabla 2, se verifica también que el $\mathrm{R}^{2}$ ajustado registró una ligera mejora $(0,951191$ para 0,953797$)$ y el F-estadístico también se presenta más significativo (50,29321 para 62,21835), indicando mejora en el coeficiente de determinación y mayor capacidad del modelo para predecir los cambios en la variable RPFD.

Aplicando el test de White, para identificar algún problema de heteroscedasticidad, se obtuvo como resultado los datos constantes de la Tabla 4. Los resultados presentados demuestran que el modelo proyectado en la Tabla 3 no presenta problemas de heteroscedasticidad. El rechazo de la existencia de heteroscedasticidad está indicada por la probabilidad del F-Estadístico, que es significativa, esto es, 0,723421, presentándose, según expresa Gujarati (2006), muy por encima del límite de 0,05 (Prob[F-estadístico] > 0,05). Considerando la no existencia de heteroscedasticidad, se verifica que esta condición no impide la aceptación del modelo para explicar la variación del RPFD en esta muestra. 
Tabla 4: Análisis del RPFD del IBOVESPA - Proyección del error de los términos de las variables del modelo final (Test de White)

\begin{tabular}{|c|c|c|c|c|}
\hline \multicolumn{5}{|c|}{ Dependent Variable: ERRO_RPFD^2 } \\
\hline \multicolumn{2}{|c|}{ Method: Panel Least Squares } & \multicolumn{3}{|c|}{ Sample: 20032005} \\
\hline \multicolumn{2}{|c|}{ Cross-sections included: 29} & \multicolumn{3}{|c|}{ Total panel (balanced) observations: 87} \\
\hline Variable & Coefficient & Std. Error & t-Statistic & Prob. \\
\hline $\mathrm{C}$ & $1.50 \mathrm{E}-05$ & $2.69 \mathrm{E}-05$ & 0.556407 & 0.5794 \\
\hline ENDTO & $2.86 \mathrm{E}-05$ & 0.000100 & 0.286321 & 0.7753 \\
\hline ENDTO $^{\wedge} 2$ & $-4.29 \mathrm{E}-05$ & 8.89E-05 & -0.483058 & 0.6303 \\
\hline R-squared & 0.007679 & \multicolumn{2}{|l|}{ F-statistic } & 0.325015 \\
\hline Adjusted R-squared & -0.015948 & \multicolumn{2}{|c|}{ Prob(F-statistic) } & 0.723421 \\
\hline Durbin-Watson stat & 0.600211 & & & \\
\hline
\end{tabular}

Siguiendo el proceso de validación del modelo, es necesario analizar la estadística "d" de Durbin-Watson, para identificar la posibilidad de la existencia de autocorrelación entre las variables del modelo. Analizando la tabla de valores aceptados para este test, en la actual regresión, se concluye que el valor del test "d" debería presentarse cerca al valor de 2,00, no presentarse inferior al 1,6, ni tampoco presentarse por encima del 2,4 (GUJARATI, 2006). Así, a partir de los dados presentados en la Tabla 3, se identifica un valor de 2,369109 relativo a la estadística "d" de Durbin-Watson, que se presenta dentro del límite establecido, comprobando que no existe autocorrelación entre las variables explicativas del modelo.

Considerando la corrección del modelo, por los resultados de los tests realizados, se concluye que la variación del RPFD en la muestra de empresas del IBOVESPA, durante el período analizado, es explicada próximamente al $95 \%$ por la variación del ratio del Endeudamiento (ENDTO), afirmación comprobada por el coeficiente de determinación $\left(\mathrm{R}^{2}\right.$ ajustado) de 0,953797. La ecuación final que se puede utilizar para determinar la variación del RPFD en la muestra IBOVESPA es: RPFD $=0,023507-0,018995 E N D T O+\alpha_{i}+e_{i}$

Conocido el modelo final que explica la variación del RPFD en la muestra de empresas brasileñas estudiadas, es importante presentar las siguientes consideraciones:

a. Para el ratio ENDTO, se esperaba en la hipótesis formulada (Hipótesis 1), que su relación con la variable RPFD fuese $\beta_{1}<0$. Esta suposición se ha confirmado una vez que su parámetro estimado en el modelo final es -0,018995, y por lo tanto, menor que cero; y

b. Interpretando el termino del ratio ENDTO y su parámetro estimado, se verifica que un aumento de una unidad decimal $(0,1)$ en dicho ratio, disminuirá el RPFD en 0,001899 .

Se puede concluir que las empresas estudiadas pueden no estar reconociendo un valor mayor de Pasivos Fiscales Diferidos cuando estuvieren con un ratio endeudamiento más elevado, justamente para no empeorar este ratio, es decir, no aumentar un endeudamiento que ya puede encontrarse en niveles por encima de lo aceptable.

Con este análisis, se pudo comprobar que en las empresas brasileñas del IBOVESPA, escogidas como muestra en el estudio, durante el periodo analizado, la variación del nivel de Pasivos Fiscales Diferidos (RPFD) se pudo explicar por la variación de su Endeudamiento. 


\subsection{Análisis de la Relación entre el RPFD y las Variables Explicativas en la Muestra de Empresas Españolas del IBEX-35}

A partir de los datos obtenidos de los estados financieros de la muestra de empresas españolas que integran el IBEX-35, se han calculado los ratios que han sido utilizados para confrontar las hipótesis formuladas a partir del modelo econométrico general. Para dar a conocer las características básicas de las variables estudiadas, se presenta a continuación la Tabla 5, la estadística descriptiva de los ratios analizados.

Tabla 5: Estadística Descriptiva II - variables de la muestra de empresas del IBEX-35

\begin{tabular}{|c|c|c|c|c|c|c|c|}
\hline $\begin{array}{c}\text { Descriptive } \\
\text { Statistic }\end{array}$ & RPFD & ENDTO & LC & LG & RF & T1 & T2 \\
\hline Mean & 0,006066 & 0,525927 & 1,106174 & 0,948541 & 0,192782 & 5,324748 & 6,545799 \\
\hline Median & 0,000845 & 0,552749 & 1,032669 & 0,878834 & 0,163211 & 5,762346 & 6,498469 \\
\hline Maximum & 0,042628 & 0,864025 & 3,267606 & 3,338624 & 0,857621 & 6,674857 & 7,711784 \\
\hline Minimum & 0,000000 & 0,150289 & 0,093164 & 0,116915 & $-0,761459$ & 1,462398 & 5,421837 \\
\hline Std. Dev. & 0,008913 & 0,169419 & 0,592394 & 0,594620 & 0,221592 & 1,087621 & 0,503685 \\
\hline Skewness & 1,511852 & $-0,368559$ & 0,612824 & 1,011357 & $-0,502741$ & $-1,467277$ & 0,281568 \\
\hline Kurtosis & 4,967502 & 2,266156 & 3,708238 & 4,788373 & 8,279459 & 5,329197 & 2,893940 \\
\hline Jarque-Bera & 47,175210 & 3,921782 & 7,263821 & 26,424990 & 104,703400 & 50,883290 & 1,190344 \\
\hline Probability & 0,000000 & 0,140733 & 0,026466 & 0,000002 & 0,000000 & 0,000000 & 0,551468 \\
\hline Observations & 87 & 87 & 87 & 87 & 87 & 87 & 87 \\
\hline
\end{tabular}

A partir de la observación de la estadística descriptiva en la Tabla 5, se identifica que la variable dependiente, el RPFD, no se caracteriza como una distribución normal. De manera semejante que en la muestra IBOVESPA, se identifica una significativa diferencia entre los niveles de pasivos fiscales diferidos a partir de los valores mínimos, máximos y medianos de la variable RPFD, lo que sugiere la adopción de prácticas contables distintas en las empresas.

De las variables explicativas, la variable $\mathrm{T} 2$ tiene una probabilidad superior al $55 \%$ $(0,551468)$ y la variable ENDTO una probabilidad del $14 \%(0,140733)$ para que puedan ser consideradas como variables con distribución normal. En lo que se refiere a las demás variables explicativas, ninguna de ellas puede ser considerada como si fuera una distribución normal. Sin embargo, esto no impide su utilización para el análisis y la estimación en el modelo econométrico propuesto.

A continuación, presentase el análisis preliminar de la relación que puede existir entre el RPFD y el conjunto de las variables explicativas en la muestra de las empresas españolas del IBEX-35. El Modelo Econométrico General propuesto para el análisis del conjunto de variables de las empresas del IBOVESPA también se ha utilizado para el presente análisis. 
Para la estimación del modelo general, se realizó una regresión múltiple por mínimos cuadrados ordinarios, con los datos agrupados en panel, y procediendo la estimación por efecto fijo, obteniéndose el resultado presentado en la Tabla 6.

La regresión estimada presenta un coeficiente de determinación $\left(\mathrm{R}^{2}\right.$ ajustado) igual a 0,806516 , esto es, la variación de la variable dependiente (RPFD) puede ser explicada en un porcentaje próximo al $81 \%$ por el conjunto de las variables explicativas que componen el modelo econométrico. Sin embargo, ninguna de las variables explicativas presenta una probabilidad significativa de real explicación en el modelo.

Observando la Tabla 6, se verifica que las variables LC, LG, RF y T2 presentan una contribución muy débil en la explicación del modelo, porque la probabilidad de su pequeña significación para la explicación de la variación de la variable dependiente es respectivamente: $0.7389,0.7856,0.8734$ y 0.8181 . Esto significa que la probabilidad de aceptación de la hipótesis nula en el poder de predicción de estas variables en el modelo está por encima del $70 \%$.

En esta situación es necesario quitar del modelo estas variables explicativas para una nueva estimación. Como consecuencia se aceptan las hipótesis nulas $\mathrm{H}_{0.2}, \mathrm{H}_{0.3}, \mathrm{H}_{0.4}, \mathrm{y} \mathrm{H}_{0.6}$, rechazándose las hipótesis que afirmaban que la variación del Ratio de los Pasivos Fiscales Diferidos (RPFD) de la muestra de empresas españolas del IBEX-35 tenía relación significativa con la variación de los ratios LC, LG, RF y T2.

Tabla 6: Regresión múltiple del RPFD y todas las variables explicativas de la muestra IBEX-35

\begin{tabular}{c|c|c|c|c}
\hline \multicolumn{5}{l}{ Dependent Variable: RPFD } \\
$\begin{array}{c}\text { Method: Panel Least Squares } \\
\text { Cross-sections included: } 29\end{array}$ & \multicolumn{2}{l}{ Tample: 20032005} \\
\hline Variable & Coefficient & Std. Error & t-Statistic & Prob. \\
\hline ENDTO & -0.013615 & 0.013451 & -1.012229 & 0.3161 \\
\hline LC & 0.000613 & 0.001831 & 0.335054 & 0.7389 \\
\hline LG & 0.000998 & 0.003651 & 0.273489 & 0.7856 \\
\hline RF & -0.000566 & 0.003538 & -0.160107 & 0.8734 \\
\hline T2 & 0.003041 & 0.002263 & 1.343533 & 0.1849 \\
\hline C & 0.001927 & 0.008332 & 0.231221 & 0.8181 \\
\hline R-squared & -0.017091 & 0.055898 & -0.305753 & 0.7610 \\
\hline Durbin-Watson stat & Cross-section fixed (dummy variables) & & 11.54353 \\
\hline
\end{tabular}

En la nueva estimación se mantuvo como variables explicativas el ENDTO y T1 (valor neto de la Cifra de Negocios), buscando así, mejorar el poder de predicción del modelo. En la Tabla 7, se puede observar el resultado de la nueva estimación del modelo. 
Evidencia de Utilización Oportunista de Normas Contables en El Reconocimiento de

Pasivos Fiscales Diferidos en Empresas Brasileñas y Españolas

Tabla 7: Regresión múltiple del RPFD y las variables ENDTO y T1 de la muestra IBEX-35

\begin{tabular}{c|c|c|c|c}
\hline \multicolumn{5}{l}{ Dependent Variable: RPFD } \\
\hline $\begin{array}{l}\text { Method: Panel Least Squares } \\
\text { Variable }\end{array}$ & \multicolumn{1}{l}{ Sample: 2003 2005 } \\
\hline Cross-sections included: 29 & Coefficient & Std. Error & t-Statistic & \multicolumn{1}{l}{ Prob. } \\
\hline ENDTO & -0.014408 & 0.011329 & -1.271844 & 0.2087 \\
\hline T1 & 0.002823 & 0.002116 & 1.334222 & 0.1875 \\
\hline C & -0.001386 & 0.011380 & -0.121781 & 0.9035 \\
\hline R-squared & Cross-section fixed (dummy variables) & & 13.97688 \\
\hline Adjusted R-squared & 0.882181 & F-statistic & & 0.000000 \\
\hline Durbin-Watson stat & 0.819064 & Prob(F-statistic) & \\
\hline
\end{tabular}

Los resultados obtenidos con la nueva estimación del modelo, revelan que la variación del RPFD puede ser explicada próximamente al $82 \%$ por la variación de las variables explicativas ENDTO y T1. Esto se comprueba de manera parcial por el $\mathrm{R}^{2}$ ajustado de la regresión que es 0,819064. Comparándose los datos de la Tabla 6 con los de la Tabla 7, se percibe que el $\mathrm{R}^{2}$ ajustado registró una ligera mejora $(0,806516$ para 0,819064) y el F-estadístico también se presenta más significativo (11,54353 para 13,97688), indicando mejora en el coeficiente de determinación y mayor capacidad del modelo para predecir el RPFD.

Sin embargo, permanece relevante la posibilidad de no contribución significativa de las variables ENDTO y T1 en la explicación de la variación del RPFD, con probabilidades respectivas de 0,2087 y 0,1875. Así, no es posible afirmar que estas variables expliquen adecuadamente la variación del ratio de Pasivos Fiscales Diferidos en la muestra de empresas españolas, a pesar de observarse la tendencia de relación entre el RPFD y estas dos variables. Para identificar algún aspecto que pueda estar perjudicando el poder de predicción de las variables ENDTO y T1 en el modelo, se realizó el test White para identificar problemas de heteroscedasticidad entre las variables, según se presenta en la Tabla 8.

Tabla 8: Análisis del RPFD del IBEX-35 - Proyección del error de los términos de las variables del modelo (Test de White)

\begin{tabular}{|c|c|c|c|c|}
\hline \multicolumn{5}{|c|}{ Dependent Variable: ERRO_RPFD^2 } \\
\hline \multicolumn{2}{|c|}{ Method: Panel Least Squares } & \multicolumn{3}{|c|}{ Sample: 20032005} \\
\hline \multicolumn{2}{|c|}{ Cross-sections included: 29} & \multicolumn{3}{|c|}{ Total panel (balanced) observations: 87} \\
\hline Variable & Coefficient & Std. Error & t-Statistic & Prob. \\
\hline $\mathrm{C}$ & $1.12 \mathrm{E}-05$ & $5.66 \mathrm{E}-05$ & 0.198752 & 0.8429 \\
\hline ENDTO & -0.000826 & 0.000160 & -5.151866 & 0.0000 \\
\hline $\mathrm{T} 1$ & 0.000105 & $2.65 \mathrm{E}-05$ & 3.974546 & 0.0002 \\
\hline $\mathrm{ENDTO}^{\wedge} 2$ & 0.000692 & 0.000156 & 4.433086 & 0.0000 \\
\hline $\mathrm{T} 1^{\wedge} 2$ & $-1.15 \mathrm{E}-05$ & $2.84 \mathrm{E}-06$ & -4.062362 & 0.0001 \\
\hline R-squared & 0.355016 & \multicolumn{2}{|l|}{ F-statistic } & 11.28371 \\
\hline Adjusted R-squared & 0.323553 & \multicolumn{2}{|c|}{ Prob(F-statistic) } & 0.000000 \\
\hline Durbin-Watson stat & 1.334032 & & & \\
\hline
\end{tabular}


Los resultados obtenidos en la Tabla 8 revelan que el modelo proyectado en la Tabla 7 presenta problemas de heteroscedasticidad, pues la probabilidad de rechazo de esta posibilidad, indicada por la probabilidad del F-Estadístico, es igual a 0,00, estando, según expresa Gujarati (2006), por debajo del límite de 0,05 (Prob[F-estadístico] > 0,05).

Por la existencia de heteroscedasticidad en el modelo, es necesario corregir el problema, y para tanto se especificará el modelo con la inclusión del parámetro de covarianza y errores típicos para problemas de heteroscedasticidad, que ha sido desarrollado por White.

A través de la estimación del modelo con la inclusión de ese parámetro, esto es, la matriz de varianzas y covarianzas de los estimadores consistente frente a problemas de heteroscedasticidad (matriz de White), se obtuvo el resultado presentado en la Tabla 9.

Tabla 9: Proyección del RPFD del IBEX-35 con la inclusión de la matriz de White

\begin{tabular}{l|c|l|l|l}
\hline \multicolumn{5}{l}{ Dependent Variable: RPFD } \\
$\begin{array}{c}\text { Method: Panel Least Squares } \\
\text { Cross-sections included: 29 }\end{array}$ & \multicolumn{1}{l}{ Sample: 2003 2005 } \\
\hline Variable & Coefficient & Std. Error & t-Statistic & \multicolumn{1}{l}{ Prob. } \\
\hline ENDTO & -0.014408 & 0.000572 & -25.20475 & 0.0000 \\
\hline T1 & 0.002823 & 0.001207 & 2.338939 & 0.0229 \\
\hline C & -0.001386 & 0.006047 & -0.229192 & 0.8196 \\
\hline R-squared & Cross-section fixed (dummy variables) & \\
\hline Adjusted R-squared & 0.882181 & F-statistic & 13.97688 \\
\hline Durbin-Watson stat & 0.819064 & Prob(F-statistic) & 0.000000 \\
\hline
\end{tabular}

La inclusión del parámetro adicional (matriz de White) en la proyección posibilitó que las variables explicativas ENDTO y T1 presentasen una mejora significativa en su poder de explicación en el modelo. Mirando hacia los datos de la Tabla 9 se observa que la probabilidad de que estas dos variables no contribuyan para la explicación de la variable dependiente está muy cerca al valor cero, es decir, las variables ENDTO y T2, a partir de la nueva estimación presentan alta probabilidad de predicción en el modelo.

El ratio endeudamiento (ENDTO) es el más significativo para la explicación de la variación del RPFD, presentando un t-estadístico $=-25,20475$. Ya el ratio T1, que presenta un t-estadístico $=2,338939$, tiene un menor poder de predicción, pero es también importante para la explicación del modelo. Por fin, es importante considerar que la estimación presentada con la inclusión de la matriz de White, mantiene el mismo coeficiente de determinación $\left(\mathrm{R}^{2}\right.$ ajustado $\left.=0,819064\right)$ y el mismo F-Estadístico (13,97688), registrado anteriormente.

Siguiendo el proceso de validación del modelo, se analiza la estadística "d" de DurbinWatson, para identificar la posibilidad de la existencia de autocorrelación entre variables del modelo. Analizando la tabla de valores aceptados para este test, en la actual regresión, se concluye que el valor del test "d" debería presentarse cerca del valor 2,00, no presentarse inferior al valor 1,6 ni tampoco presentarse muy por encima del 2,3 (Gujarati, 2006).

Basándose en los datos presentados en la Tabla 9, se identifica un valor de 3,479645 
relativo a la estadística "d" de Durbin-Watson, que presentase un tanto por encima del límite establecido. Sin embargo, también hay que considerar que, en las estimaciones de modelos con dos variables explicativas y con un número de observaciones cerca de las noventa, como es el caso de la actual regresión, cuando la estadística "d" de Durbin-Watson se presenta superior al valor 1,703, no habrá ninguna evidencia de correlación serial positiva de primer orden (Gujarati, 2006). En este contexto, se acepta el modelo para predecir la explicación de la variable RPFD en la muestra de empresas españolas del IBEX-35.

Considerando la corrección del modelo por los resultados de los tests realizados, se concluye que la variación del RPFD en la muestra de empresas IBEX-35, durante el período analizado, es explicada próximamente al $82 \%$ por la variación de los ratios de Endeudamiento y Tamaño 1. Esta afirmación puede ser comprobada por el coeficiente de determinación $\left(\mathrm{R}^{2}\right.$ ajustado) de 0,819064 del modelo y la ecuación final utilizada para explicar la variación del RPFD en la muestra es: RPFD $=-0,001386-0,014408 E N D T O+0,002823 t 1+\alpha_{i}+e_{i}$

Conocido el modelo final que explica la variación del RPFD en la muestra de empresas españolas, es importante presentar las siguientes consideraciones:

a. Para el ratio ENDTO, se esperaba en la hipótesis formulada, que su relación con la variable RPFD fuese $\beta_{1}<0$. Esta suposición se ha confirmado una vez que su parámetro estimado en el modelo final es $-0,014408$ y por lo tanto, menor que cero;

b. Interpretando el término del ratio ENDTO y su parámetro estimado, se verifica que un aumento de una unidad decimal $(0,10)$ en dicho ratio, disminuirá el RPFD en $0,00144, \mathrm{y}$

c. Para el ratio $\mathrm{T} 1$, se esperaba en la hipótesis formulada, que su relación con la variable RPFD fuese $\beta_{5} \neq 0$. Esta suposición se ha confirmado, una vez que su parámetro estimado en el modelo es 0,002823 , esto es, mayor que cero, por lo tanto, diferente de cero; $y$

d. Interpretando el término del ratio T1 y su parámetro de estimación, se verifica que un aumento de un aumento de $1 \%$ en dicho ratio, aumentará el RPFD en $0,000028 \%$.

A partir de los resultados se percibe que los gerentes de las empresas estudiadas pueden no reconocer un valor más significativo de Pasivos Fiscales Diferidos cuando el ratio de endeudamiento de la empresa está muy alto. Dicho reconocimiento podría empeorar este ratio, aumentando un endeudamiento que ya puede estar en niveles por encima de lo aceptable.

Los resultados de la regresión demuestran también que en las empresas con mayor Tamaño 1, mayor se presentará el ratio de Pasivos Fiscales diferidos, esto es, las empresas con mayor valor de Cifra Neta de Negocios demuestran mayor tendencia en reconocer pasivos diferidos por Impuesto sobre Beneficios.

Con el análisis realizado en este apartado, se pudo demostrar que en la muestra de empresas del IBEX-35, escogidas para el presente estudio, durante el periodo analizado, la variación del Ratio de Pasivos Fiscales Diferidos (RPFD) se pudo explicar por la variación de su Endeudamiento y de su tamaño calculado a partir del valor de sus Ingresos Netos. 


\section{CONSIDERACIONES FINALES}

A partir del estudio empírico realizado ha sido posible obtener algunas conclusiones significativas sobre el nivel de reconocimiento de Pasivos Fiscales Diferidos en las muestras de empresas estudiadas.

A través del análisis realizado en la muestra de empresas brasileñas del IBOVESPA se ha concluido, por la estimación final del modelo, que los gestores pueden estar reconociendo un valor menor de Pasivos Fiscales Diferidos cuando las empresas presenten un ratio de endeudamiento más elevado, justamente para no empeorar este ratio, es decir, no aumentar un endeudamiento que ya puede encontrarse en niveles por encima de lo aceptable.

Cuando este análisis se ha realizado en la muestra de empresas españolas del IBEX-35, los resultados se han presentado semejantes en lo que se refiere al endeudamiento. A partir de los resultados de la regresión se percibe que los gestores de las empresas españolas también pueden no reconocer un valor más significativo de Pasivos Fiscales Diferidos cuando el ratio de endeudamiento de la empresa esté muy elevado. En estas ocasiones, dicho reconocimiento podría empeorar este ratio, es decir, aumentaría un endeudamiento que ya puede encontrarse en niveles por encima de lo aceptable por los directivos de la empresa.

Sin embargo, según el modelo proyectado, en la muestra de empresas del IBEX-35, el tamaño también presenta relación con el Ratio de Pasivos Fiscales diferidos. Los resultados de la regresión demuestran en las empresas con mayor Tamaño 1, mayor se presentará el ratio de Pasivos Fiscales diferidos, esto es, las empresas con mayor valor de Cifra Neta de negocios demuestran mayor tendencia en reconocer pasivos diferidos por Impuesto de Sociedades.

Esta parte del estudio es novedosa con respecto a otras investigaciones realizadas y que han sido referidas en el estudio. Sin embargo, en las dos muestras investigadas, los resultados obtenidos señalan que los gestores de las empresas pueden estar aprovechándose de la discrecionalidad de las normas contables para reconocer un nivel mayor o menor de pasivos fiscales diferidos, o no reconocer dichos pasivos. Los resultados obtenidos remiten hacia una fuerte "evidencia de adopción oportunista de normas contables en el reconocimiento de pasivos fiscales diferidos", situación caracterizada por manipulación contable.

El estudio debe tener seguimiento, con la realización de investigaciones semejantes en otras muestras de empresas y en períodos más recientes, buscando así evaluar la evolución de la práctica contable en este tema y en otros temas relacionados con la manipulación contable. Es importante señalar que la continuación de este estudio es un planteamiento del grupo de investigadores españoles y brasileños, involucrados en la investigación del tema.

\section{REFERENCIAS}

ALONSO C., I., NEVADO P., D. y NÚÑEZ C., M. La reforma contable y la relación contabilidadfiscalidad. Revista Partida Doble, n... 147, p. 18-33, 2003.

CASTILLO, J. Impuesto sobre beneficios (NIC 12), In: Normas Internacionales de Contabilidad (NIC/NIIF). Barcelona, Ediciones Gestión 2000, 2004.

CFC - CONSELHO FEDERAL DE CONTABILIDADE. Resolução CFC nº 998/2004 - NBC 
T 19.2 - Tributos sobre lucros. Disponible en:http://www.cfc.org.br/, acceso en 06/05/2009.

CPC - COMITÊ DE PRONUNCIAMENTOS CONTÁBEIS. Pronunciamento Técnico CPC 32 - Tributos sobre o Lucro. Disponible en: http://www.cpc.org.br/pdf/CPC_32.pdf. Acceso en: $29 / 01 / 2010$.

CVM - COMISSÃO DE VALORES MOBILIÁRIOS. Deliberação CVM No 273/1998. Brasília, 1998. Disponível em: http://www.cvm.gov.br/, Acesso em: 06/05/2009.

ESPAÑA - REAL DECRETO 1.643/1.990, por el que se aprueba el Plan General de Contabilidad.

ESPAÑA - REAL DECRETO 1514/2007, por el que se aprueba el Plan General de Contabilidad.

FASB - FINANCIAL ACCOUNTING STANDARDS BOARD. Statement of financial accounting standards (SFAS) $N^{\circ}$ 109: Accounting for Income Taxes. Stamford, 1992.

GALLEGO A., I. Análisis del SFAS 109 y consideración contable de los impuestos anticipados: una interpretación del caso español. Centro de Estudios Financieros, Revista de Contabilidad y Tributación, n. 171, p. 127-154, 1997.

GARCÍA-AYUSO C., Manuel. y ZAMORA R., Constancio. Análisis de dos factores determinantes en el reconocimiento de créditos por pérdidas fiscales en las empresas españolas. Revista Española de Financiación y Contabilidad, v. XXXII, nº 117, p. 395-429, 2003.

GORDON, E. A.; JOOS, P. R. Unrecognized deferred taxes: evidence from the U. K., The Accounting Review, v. 79, n. 1, p. 97-124, January 2004.

GUJARATI, Damodaran. N. Econometria básica. 4a edição. Elsevier, Rio de Janeiro, 2006.

HENDRIKSEN, E. S.; VAN BREDA, M. F. Teoria da contabilidade. São Paulo, Atlas, 1999.

HILL, R. Carter; GRIFFITHS, William E.; JUDGE, George G. Econometria. 2a Ed., São Paulo, Saraiva, 2003.

ICAC - INSTITUTO DE CONTABILIDAD Y AUDITORÍA DE CUENTAS. Norma Internacional de Contabilidad $n^{\circ} 12$ (NIC 12) - Impuesto sobre las Ganancias. Disponible en: http://www.icac.mineco.es/, acceso en: 20 oct. 2005.

ICAC - INSTITUTO DE CONTABILIDAD Y AUDITORIA DE CUENTAS. Resolución del 9/octubre/1997, sobre algunos aspectos de la norma de valoración $16^{\mathrm{a}}$ del Plan General de Contabilidad.

ICAC - INSTITUTO DE CONTABILIDAD Y AUDITORIA DE CUENTAS. Resolución del 15 de marzo de 2002, por la que se modifica parcialmente la de fecha 8 de octubre de 1997 sobre algunos aspectos de la norma de valoración decimosexta del Plan General de Contabilidad.

IUDÍCIBUS, Sérgio de. Teoria da contabilidade. $8^{\text {a }}$ Ed., São Paulo, Atlas, 2006.

KRONBAUER, Clóvis A.; MORENO R. José. Tratamento contábil dos tributos sobre o lucro: 
um estudo comparativo entre as normas brasileiras da CVM e do CFC e a norma internacional de contabilidade $\mathrm{n}^{\circ} 12$ do IASB. In: CONVENÇÃO DE CONTABILIDADE DO RIO GRANDE DO SUL, 10. 2008. Anais... Bento Gonçalves, CRC-SP, 2005.

KRONBAUER, Clóvis A.; ALVES, Tiago W. Fatores determinantes no reconhecimento de ativos fiscais diferidos numa amostra de empresas do Ibovespa no período de 2003/2005. In; ENCONTRO DA ASSOCIAÇÃO NACIONAL DE POS-GRADUAÇÃO E PESQUISA EM ADMINISTRAÇÃO - ENANPAD, 32, 2008. Anais... Rio de Janeiro, ANPAD, 2008.

LABATUT S., Gregorio. Contabilidad y fiscalidad del resultado empresarial. Instituto de Contabilidad y Auditoría de Cuentas, Ministerio de Economía y Hacienda. Madrid, 1992.

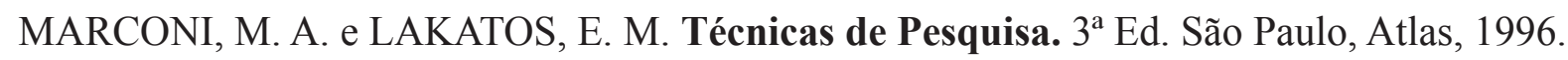

MILLER, G. S.; SKINNER, D. J. Determinants of the valuation allowances for deferred tax assets under SFAS n 109, The Accounting Review, v. 73, n. 2, p. 213-233, April 1998.

MORENO R., José. Contabilidad y fiscalidad: diferencias entre resultado contable y base imponible en el nuevo impuesto sobre sociedades. Sevilla, Universidad de Sevilla, 1997.

MORENO R., José; LOPES H., Diego; KRONBAUER, Clóvis A.; SOUZA, Marcos A. La activación de las diferencias temporales positivas en empresas cotizadas españolas: un estudio empírico. In: CONGRESSO INTERNACIONAL DA ASSOCIAÇÃO NACIONAL DE PROGRAMAS DE PÓS-GRADUAÇÃO EM CIÊNCIAS CONTÁBEIS - ANPCONT \&, IAAER, 3, 2009. Anais... São Paulo, IAAER-ANPCONT, 2009.

OPPENHEIM, A. N. Questionnaire design, interviewing and attitude measurement. London, Pinter, 1993.

WASSERMAN, Cláudio. O Ativo fiscal diferido no sistema financeiro nacional: análise e proposta de contabilização. Dissertação (Mestrado em Controladoria e Contabilidade). Faculdade de Economia, Administração e Contabilidade, Departamento de Contabilidade e Atuária da USP. São Paulo, FEA/USP, 2004.

WOOLDRIDGE, J. M. Introdução à econometria: uma abordagem moderna. $2^{\mathrm{a}}$ Ed., São Paulo, Pioneira Thomson Learning, 2006. 


\section{DADOS DOS AUTORES:}

\section{CLÓVIS ANTONIO KRONBAUER}

Universidade do Vale do Rio dos Sinos, Centro de Ciências Econômicas.

Av. Unisinos, 950 - Cristo Rei

93022-000 - Sao Leopoldo, RS - Brasil

\section{JOSÉ MORENO ROJAS}

Facultad de Turismo y Finanzas

Avda. San Francisco Javier, s/n

41015 - Sevilla (España)

\section{ERNANI OTT}

Universidade do Vale do Rio dos Sinos, Área Econômica, Área de

Conhecimento e Aplicação Contabilidade Custos e Finanças.

Av. Unisinos, 950 - Cristo Rei

93022-000 - Sao Leopoldo, RS - Brasil - Caixa-postal: 275

\section{MARCOS ANTONIO SOUZA}

Universidade do Vale do Rio dos Sinos, Centro de Ciências Econômicas.

Av. Unisinos, 950 - Cristo Rei

93022-000 - Sao Leopoldo, RS - Brasil 